\title{
Prototyping a thermal walker that can walk on a hot horizontal surface with a simple gait
}

\author{
Takeru Nemoto* and Akio Yamamoto
}

\begin{abstract}
This article reports on a newly designed thermal walker that can walk on a hot horizontal surface without any motor or battery. The walker converts heat energy from the hot surface into mechanical motion by using bimetal sheets. The prototype developed in the previous study successfully demonstrated walking, but its gait was not ideal; a leg swings forward and backward a few times to achieve one step. This article designs a new walker to realize more natural walking, in which a leg simply swings forward once in each step. For this purpose, the article introduces a new design process to better estimate the swing period. Also, material used for the feet of the walker is reconsidered to realize stable continuous walking. The newly fabricated prototype has aluminum feet and can walk on a hot horizontal surface with the simple gait. The surface temperature required for walking was $110^{\circ} \mathrm{C}$, which was considerably lowered from that in the previous study, which was $170^{\circ} \mathrm{C}$.
\end{abstract}

Keywords: Bimetal, Thermal deformation, Environmental heat, Passive dynamic walker, Thermal, Walker

\section{Introduction \\ Background}

A passive dynamic walker was proposed by McGeer in 1990 to realize human-like walking $[1,2]$. Based on the passive dynamic walker, many walking systems have been developed. Typically, passive dynamic walkers have no actuator and walk down slopes utilizing the gravity. Some walking systems based on passive dynamic walkers, however, can efficiently walk on a horizontal surface, by adding actuators and batteries to the walkers [3-7]. A thermal walker, which is to be dealt with in this article, is categorized into such a kind of mechanism [8]. The walker, however, has no battery nor explicit actuator on it. Instead, it has bimetal sheets on the bottom surfaces of its cylindrical feet. A bimetal sheet is composed of two thin layers with different thermal expansion coefficients and is bent when heated. On the walker, the bimetal sheets attached to the feet convert the heat energy from

*Correspondence: t-nemoto@aml.t.u-tokyo.ac.jp

Department of Precision Engineering, The University of Tokyo, 7-3-1 Hongo, Bunkyo-ku, Tokyo 113-8656, Japan the horizontal surface into mechanical motion, which enables the walker to walk on a horizontal surface.

In the previous work on the thermal walker, a prototype successfully walked on a hot surface heated at $170^{\circ} \mathrm{C}$ [8]. By utilizing deformations of the bimetal sheets, the walker swung its body in sideways that lifted up one of its legs. The lifted leg behaved as a pendulum and thus swung forward and then landed, as the sideways swing of the body was restored. Repeating such swing motions, the thermal walker successfully walked on a hot horizontal surface. The temperature required for walking was considerably high and a lot of efforts should be made to ease the requirement of the temperature. If the temperature could be lowered in future work, the walker would be able to walk on, e.g., a hot ground surface during summer. Moreover, the mechanism would be integrated to a general bipedal robot to reduce the energy consumption; the bipedal robot would still rely on the conventional motors, but the thermal walking mechanism might help to reduce the energy consumption of the motors.

In the proposed thermal walker, the ideal, or natural walking is realized when the period of the body swing and that of the leg swing are identical; in other words, 
the period ratio is 1 . In such a condition, the leg simply steps forward as the body leans sideways. In the previous prototype, however, the period ratio was around 3. As a result, the walker swings a leg forward, backward, and then forward again for each step. Considering the future integration of the thermal walking mechanism with a general bipedal robot, for example, such complicated gait is apparently not desired; the simple gait with the period ratio of 1 should be realized.

To shorten the period ratio to realize the ratio of 1 , the leg swing period should be extended, whereas the body swing period should be shortened. However, both of them were not successfully realized in the previous work due to the constraints arising from the design process and the material. Therefore, to realize the walking with the period ratio of 1 , the walker design needs to be reconsidered.

\section{Objective of this paper}

The objective of this paper is to realize a thermal walker that can walk continuously with the period ratio of 1 . The contributions of this paper are (1) proposing a new leg swing model, (2) establishing a design process for the period ratio of 1 , and (3) revealing the effect of material on the continuity of self-induced oscillation.

First, by proposing a new leg swing model, the period of leg swing will be extended. The new model adopts a rigid-body pendulum that provides a wider range of swing period. With the new leg swing model, this paper proposes a new designing process that can realize the period ratio of 1 . This, however, requires the body swing period to be shortened, which might cause the damped oscillation due to overheating of the bimetal. To avoid overheating, the paper investigates the effect of heat conductivity of feet materials on the oscillation. With these improvements, the paper demonstrates a thermal walker that can walk continuously with the period ratio of 1 .

The remainder of this paper is structured as follows. The next section, "Thermal walker", explains the structure and the walking principle of the thermal walker more in detail. In "Design process", the new design process for the thermal walker is discussed to realize the period ratio of 1 . "Effect of materials on self-induced oscillation" investigates how the difference of heat conductivities of materials affects the performance. In "Experiments", a new prototype with the period ration of 1 is designed, and its gait is observed to discuss the effect of the leg length on the stride. Finally, "Conclusion" summarizes the work with some comments on the future direction of the work.

\section{Thermal walker}

The thermal walker can walk on a heated horizontal surface by utilizing heat energy [8]. One example is shown in Fig. 1. Its structure is simple; two legs are connected through a rotational axis. At the end of each leg, a foot with a cylindrical bottom surface is attached. When both feet are aligned, the bottom surfaces make a single cylindrical surface. Their bottom surfaces are covered with bimetal sheets, which excite oscillation when heated.

The oscillation used for the thermal walker is selfinduced oscillation of a bimetal sheet, which was originally reported in 2006 [9]. In general, self-induced oscillation is defined as "a periodic process at the expense of a non-periodic source of energy" in [10]. The self-induced oscillation of a bimetal sheet utilizes constant heating and converts the heat energy into a periodic mechanical motion using the thermal deformation of the bimetal sheet. This phenomenon was originally reported for an eccentric cylinder, as in Fig. 2. A bimetal sheet was attached to the cylinder such that its lower expansion side faces to the surface of the cylinder. When the cylinder contacts with a hot surface via the bimetal, the contacting part of the bimetal would bend locally to make a minute protrusion, as shown in the right-bottom of Fig. 2. This makes the cylinder unbalanced, and the cylinder starts to roll. The rolling changes the contact area between the bimetal and the surface, and the minute protrusion would move so as to follow the contact area. Thus, the eccentric cylinder continues rolling until its center of mass (CoM) rises too high. As CoM rises high, the gravity overcomes the rolling motion, and the cylinder changes its rolling direction. In such a manner, the eccentric cylinder automatically oscillates on the hot surface. One thing to be noticed regarding this phenomenon is that if the amplitude of the oscillation is too small or the period is

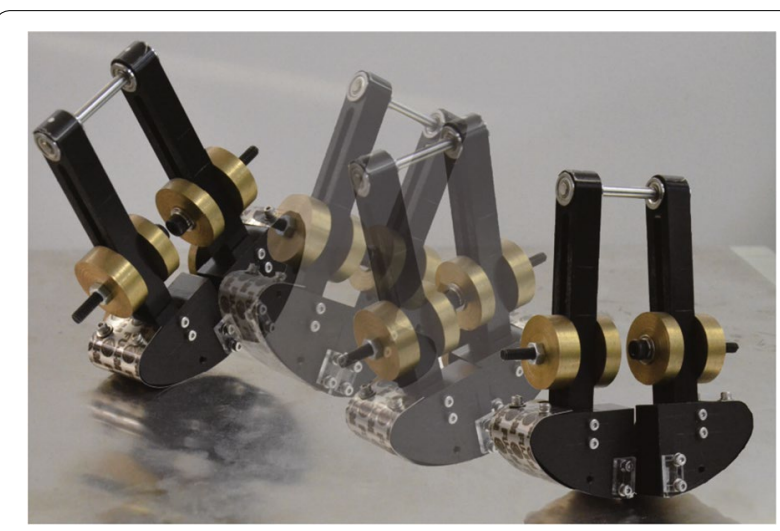

Fig. 1 Thermobot walking on a heated horizontal surface 


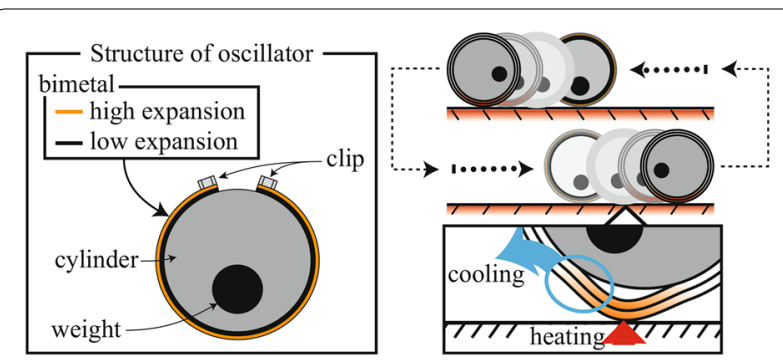

Fig. 2 Eccentric cylinder with bimetal sheet and its self-oscillation on a hot plate (From [8], (2015 IEEE)

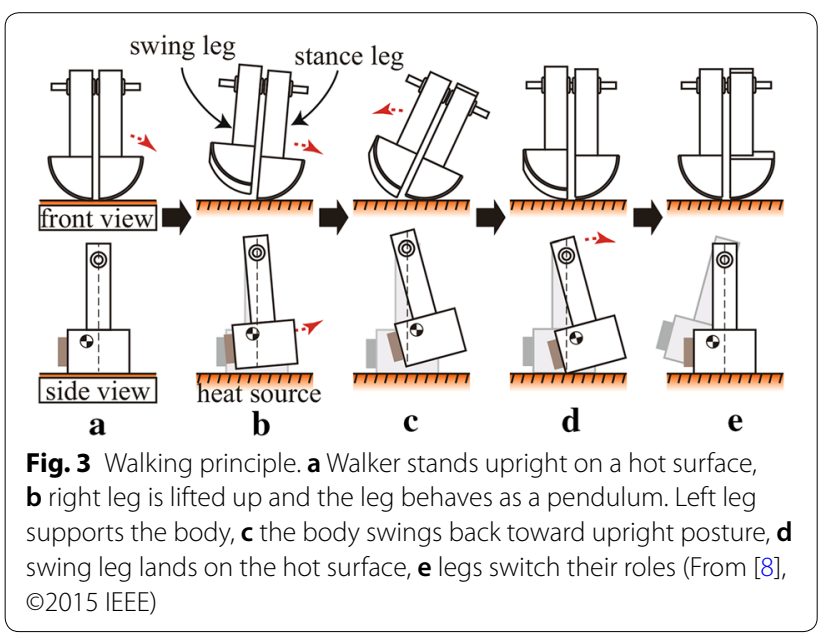

too short, the bimetal sheet is overheated and the oscillation will be damped and will eventually stop.

The self-induced oscillation can be used as an energy source of motion systems. In [11, 12], a linear actuator was developed using the self-induced oscillation. In the developed actuator, an eccentric cylinder integrated in the actuator continuously hit the side wall of the actuator body. Then, by the impact of the hitting, the whole actuator body placed on a heated frictional surface moved step-by-step. However, applications of the self-induced oscillation had not been extensively studied until the thermal walker was proposed in 2015.

The thermal walker realizes walking by converting the self-induced oscillation into pendulum movement of the swing leg, as in Fig. 3. When the walker is arranged upright on a hot surface, the bimetal sheets on the bottom of its feet excite the self-induced oscillation, which makes the walker body roll in sideways. Then, the rolled walker stands on one leg (stance leg), while the other leg is lifted up to be a swing leg. The swing leg swings forward like a pendulum, since the center of mass (CoM) of the swing leg is shifted backward. A moment later, the body swings back toward the upright posture, and then the swing leg lands on the hot surface and becomes a new stance leg. The former stance leg is now lifted up to be a new swing leg. The walker repeats the same motion to walk continuously.

\section{Period ratio}

The gait of the thermal walker can be analyzed considering the period ratio of the two swings [8]. To evaluate the period ratio, we assume that the walking motion can be decoupled into two swing motions: the body swing (selfinduced oscillation) and the leg swing (pendulum movement). The period ratio is defined as

$$
r_{p}=\frac{T_{b}}{T_{l}}
$$

where $T_{b}$ is the period of the body swing, and $T_{l}$ is the period of the leg swing.

This number also indicates how many times the swing leg swings until it lands in each step [8]. Each leg is allowed to swing for $T_{b} / 2$ during each step. The largest step is obtained if the swing leg lands on the ground, $T_{l} / 2$ after it has started to swing. Therefore, the condition for the largest step is $T_{b} / 2=T_{l} / 2$, or $r_{p}=1$. When $r_{p}=2$, $T_{b} / 2$ is equal to $T_{l}$. In this case, the stride becomes zero because $T_{l}$ is the period of full leg swing, which means that the swing leg lands on the ground at the same place as it is lifted up. When $r_{p}=3, T_{b} / 2$ is equal to $\left(1+\frac{1}{2}\right) T_{l}$, which indicates that the swing leg swings one full forward swing, one full backward swing, and one more full forward swing. This period ratio also maximizes the stride but it has unnecessary leg swings, compared to the gait for $r_{p}=1$. Similarly, any odd integer numbers of $r_{p}$ can realize the maximum step length, but as $r_{p}$ becomes larger, the gait contains more unnecessary leg swings. Therefore, it can be said that period ratio of 1 realizes the ideal walking.

The period ratio of the prototype designed in the previous work was adjustable in a range from 1.5 to 5.4 by changing the height of weights attached to its legs. Successful walking was obtained when the period ratio was 3.2 , but, at the smallest period ratio, 1.5 , the body swing was not excited and the walker did not walk. The reason would be overheat of the bimetal sheets. Smaller period ratio could be obtained when the body swing was small, which, however, made the bimetal sheets contacting with the hot surface at a limited area. Then, the bimetal and the leg would be heated up too much around the limited contact area and the minute protrusion would not be created. 

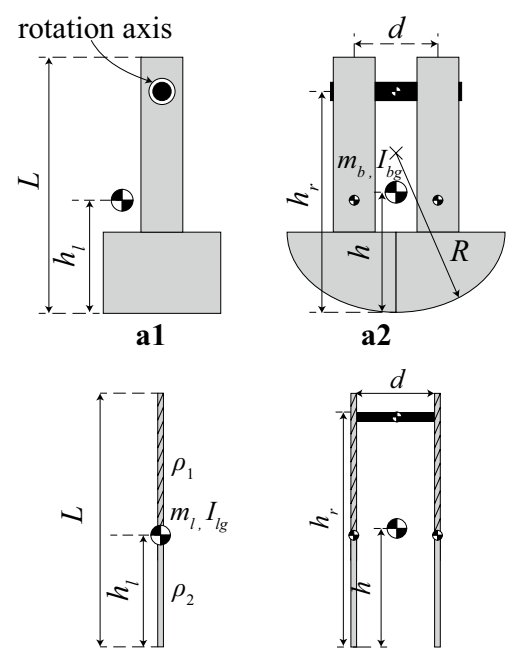

b1

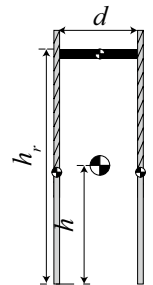

b2

Fig. 4 A schematic of a thermal walker. a1 Side view. a2 Back view. b1 A model for calculating $l_{\mathrm{g} g}$. b2 A model for calculating $l_{\mathrm{bg}}$

\section{Design process}

In this section, a design process to realize the period ratio of 1 is discussed. First, by regarding the leg as a rigid body, the period of its swing is calculated. Then, the moments of inertia of the legs are estimated by using a simplified model. After that, we introduce the design process which can robustly realize the period ratio of 1 , regardless of the height of the rotational axis.

Figure 4 shows (a) a schematic of the thermal walker and (b) a simplified model for calculating the periods. Subscript of $l, r, b$, and $g$ represent leg, rotational axis, body, and CoM, respectively.

\section{Leg swing period considering moment of inertia}

In the previous study [8], the leg was considered as a point-mass pendulum when calculating its period. It simplifies the calculation and makes a good enough estimation in some situations. For more precise estimation in broader ranges of parameters, we model the leg as a rigid-body pendulum considering the moment of inertia. By using the parameters in Fig. 4a, the leg swing period is described as

$$
T_{l}=2 \pi \sqrt{\frac{I_{l c}}{m_{l} g\left(h_{r}-h_{l}\right)}},
$$

where $I_{l c}$ is the moment of inertia of each leg about the rotation axis and is equal to $I_{l g}+m_{l}\left(h_{r}-h_{l}\right)^{2}, m_{l}$ is the weight of each leg, $g$ is the acceleration of gravity, $h_{r}$ is the height of the rotational axis from the ground, $h_{l}$ is the

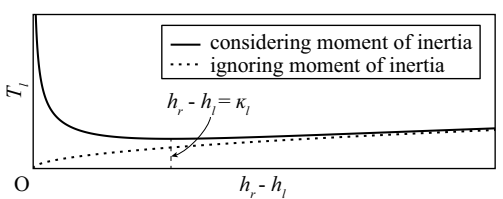

Fig. 5 Comparison of $T_{\text {/ }}$ between point-mass and rigid-body pendulum approximations

height of the leg's CoM from the ground, and $I_{l g}$ is the moment of inertia of the leg about its CoM.

Figure 5 shows the change of the leg swing period $T_{l}$ against the distance between the axis and the leg's CoM $\left(h_{r}-h_{l}\right)$. The shortest swing period is obtained at $h_{r}-h_{l}=\kappa_{l}$, where $\kappa_{l}$ is the radius of gyration of the leg and equals $\sqrt{I_{l g} / m_{l}}$. As $h_{r}-h_{l}$ gets closer to zero, the period increases to infinity. On the other hand, for $h_{r}-h_{l}$ larger than $\kappa_{l}$, the period gradually converges to the period calculated by the point-mass pendulum approximation [8] which is $2 \pi \sqrt{\left(h_{r}-h_{l}\right) / g}$. This comparison tells that the point-mass pendulum approximation in the previous work is precise enough only when $h_{r}-h_{l}$ is large enough. The plot also shows that the period $T_{l}$ is less sensitive to the change of $h_{r}$ and/or $h_{l}$ when $h_{r}-h_{l}$ is close to or larger than $\kappa_{l}$, compared to when $h_{r}-h_{l}$ is close to zero. This characteristics will be exploited in the design process described later.

\section{Calculation of period ratio}

For calculating the period of the body swing, we approximate the whole body as an eccentric cylinder. The period is then calculated using the parameters in Fig. 4a as:

$$
T_{b}=2 \pi \sqrt{\frac{m h^{2}+I_{b g}}{m g(R-h)}},
$$

where $m$ is the total weight of the walker, $h$ is the height of the CoM of the entire walker, measured from the ground, $I_{b g}$ is the moment of inertia of the walker about the walker's CoM, and $R$ is the curvature radius of the bottom surface of the feet.

The period ratio $r_{p}=T_{b} / T_{l}$ is evaluated by using Eqs. (2) and (3). Since these equations contain the moments of inertia, $I_{l g}$ and $I_{b g}$, we first need to estimate these moments. To simplify calculations, we model each leg as a thin bar, which has different constant densities above and below the CoM (see Fig. 4b1). Then $I_{l g}$ is calculated as $\frac{1}{3} m_{l}\left(L-h_{l}\right) h_{l}$.

By the same assumption, the moment of inertia of the body, $I_{b g}$, is also estimated (see Fig. 4b2). First, 
the moment of inertia of the two legs is calculated as $2 I_{l g}+2 m_{l}\left\{\left(h-h_{l}\right)^{2}+(d / 2)^{2}\right\}$, where $d$ is the distance between the two legs. However, due to the complexity of calculation, $d$ is regarded as zero in the following calculation. Then, adding the moment of inertia of the rotational axis which is calculated as $1 / 12 m_{r} d^{2}+m_{r}\left(h-h_{r}\right)^{2}$, gives the total moment $I_{b g}$. With these assumptions, the period ratio is calculated as

$$
\begin{aligned}
& r_{p}=\frac{1}{2} \sqrt{\frac{n u m}{d e n}} \\
& \text { num }=\left(h_{r}-h_{l}\right)\left[16(2+\hat{m}) h_{l}^{2}\right. \\
&+8\left\{(2-5 \hat{m}) L+6 \hat{m} h_{r}\right\} h_{l}+24 \hat{m} L^{2} \\
&\left.+12 \hat{m}^{2} h_{r}^{2}+\left(\hat{m}^{2}+8 \hat{m}+12\right) d^{2}\right] \\
& \text { den }=(2+\hat{m}) R-(2+\hat{m})\left\{2 h_{l}-\hat{m} h_{r}\right\} \\
&\left\{2 h_{l}^{2}+\left(L-6 h_{r}\right) h_{l}+3 h_{r}^{2}\right\}
\end{aligned}
$$

where $\hat{m}$ is the normalized mass and equals $m_{r} / m_{l}$.

\section{Adjusting period ratio to 1}

Here we discuss a design process to achieve $r_{p}=1$. First, $L$ needs to be decided considering a design constraint such as the desired size of the walker. For example, we adjusted $L$ to $17 \mathrm{~cm}$ to design a prototype shown in the next section.

Figure 6 shows the relationship among $h_{l}, h_{r}$, and $T_{l}$, calculated using Eq. (2). Solid contour lines show constant values of $T_{l}$. In this plot, $h_{r}$ must satisfy the following two conditions. First, $h_{r}$ which is the height of the axis, must be smaller than the total length of the walker, $L$, therefore $h_{r}<L$. Second, $h_{r}$ should be close to or larger than $h_{l}+\kappa_{l}$, since $T_{l}$ is less sensitive to the changes of $h_{r}$ and $h_{l}$ in this area, according to Fig. 5. Practically,

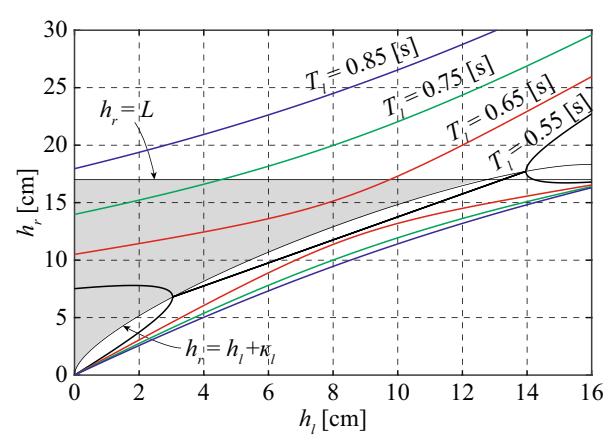

Fig. 6 Contour map of $T_{\text {l }}$ against $h_{l}$ and $h_{r}$ this is important as $h_{l}$ (and possibly $h_{r}$ ) can be deviated from the designed value due to the model simplification and manufacturing errors. The gray area in Fig. 6 satisfies these two conditions.

Now, we can pick up a point from the gray area to determine $h_{l}, h_{r}$, and $T_{l}$. For the prototype of the next section, we picked up a set of $h_{l}$ and $h_{r}$ on the contour line for $T_{l}=0.65 \mathrm{~s}$. The chosen $h_{l}$ and $h_{r}$ were 4 and $12 \mathrm{~cm}$, respectively.

Finally, by using Eq. (4), a pair of $(R, \hat{m})$ is determined to realize the period ratio of 1 . The red line in Fig. 7 indicates the $(R, \hat{m})$ relation with $h_{l}=4 \mathrm{~cm}$ and $h_{r}=12 \mathrm{~cm}$, whilst other lines show the reference relations with different values of $\left(h_{l}, h_{r}\right)$. For the prototype, we chose $R$ and $\hat{m}$ as $9 \mathrm{~cm}$ and 0.13 , respectively.

\section{Effect of materials on self-induced oscillation}

To realize continuous walking, damped oscillation must be avoided, which occurs when the bimetal sheets are overheated. The overheat can occur with a short period of the body swing $T_{b}$, which results in a small swing amplitude. As shown in Fig. 5, the leg swing period, $T_{l}$, which is almost equivalent to the body swing period $T_{b}$ when $r_{p}$ is 1 , can be set high by selecting smaller $h_{r}-h_{l}$. In such a selection, however, $T_{l}$ becomes too sensitive to a small change of $h_{r}$ and $h_{l}$. Therefore, such selection should be avoided. As a result, $T_{l}$ and $T_{b}$ should be set appropriately low with $r_{p}=1$. This imposes a risk of overheating.

To avoid overheating under such situation, the effect of feet materials on the self-induced oscillation of a bimetal sheet was investigated. In the previous study [8], polyacetal was used as the material for the feet due to ease of machining. However, heat conductivity of polyacetal is low which might have caused overheating. To examine

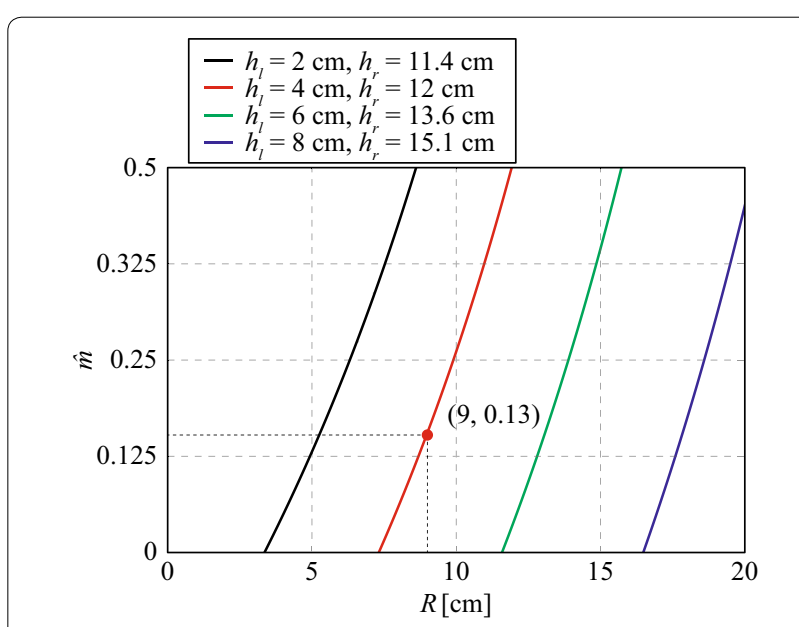

Fig. 7 Relation between $R$ and $\hat{m}$ calculated with different pairs of $h_{1}$ and $h_{r}$ that give $T_{l}=0.65 \mathrm{~s}$ 


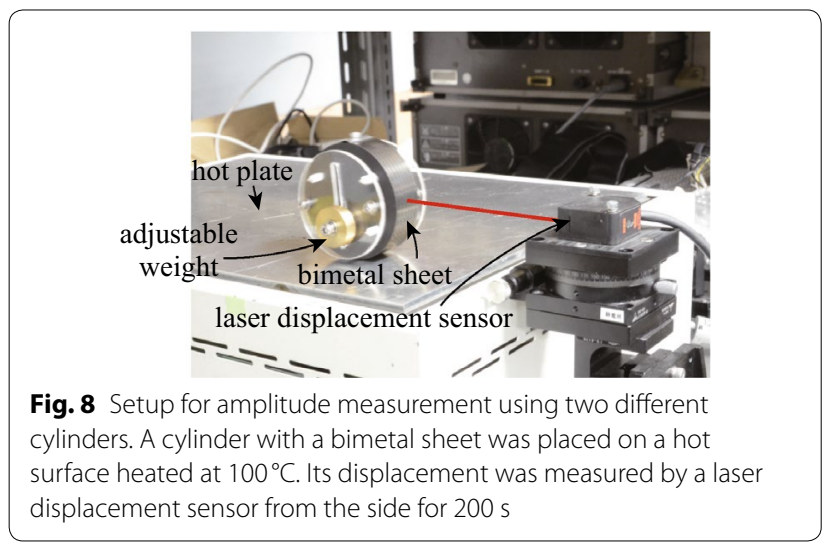

the effect of heat conductivity, the amplitude of the selfinduced oscillation was measured using cylinders made of different materials, as shown in Fig. 8. In this measurement, two materials (aluminum and MC Nylon) with considerably different heat conductivities were used for cylinders. The nominal heat conductivity of each material is 140 and $0.71 \mathrm{~W} / \mathrm{mK}$. Both cylinders had a diameter of $70 \mathrm{~mm}$ and thickness of $20 \mathrm{~mm}$. On the curved surface of each cylinder, a bimetal sheet (Engineered Materials Solutions, LLC, TRUFLEX P675R, curvature coefficient: $20.5 \times 10^{-6} \mathrm{~K}^{-1}$ ) with a thickness of $0.13 \mathrm{~mm}$ was attached. Sides of the cylinders were covered with plastic

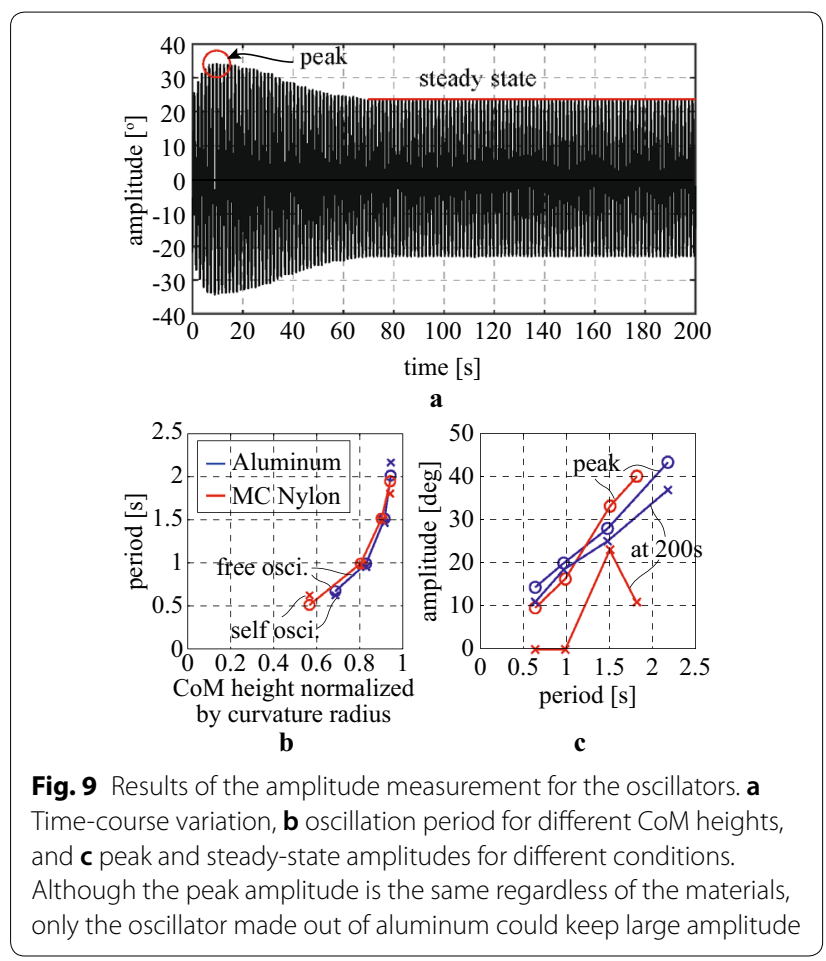

plates, on which a weight was attached to adjust the position of the CoM.

A cylinder was placed on a horizontal plate heated at $100^{\circ} \mathrm{C}$ and its position was measured using a laser displacement sensor (Omron, ZX-LD100) for $200 \mathrm{~s}$, to evaluate the variations of the swing period and the amplitude of the self-induced oscillation. Figure 9a shows an example of the measured results. The amplitude of the oscillation gradually increases in the beginning to reach the peak. Then, as the bimetal and the cylinder become hotter, the amplitude decreases until the system reaches a thermal equilibrium. The period of the oscillation, on the other hand, was found almost constant during the measurement, regardless of the change of the amplitude. Figure $9 \mathrm{~b}$ shows the oscillation period using cross marks, as a function of the CoM height normalized by the radius. The period measurement was also conducted on a regular (non-heated) horizontal surface to measure the period for free oscillation of the eccentric cylinders, which is shown using the circular markers. The plot indicates that the period of the self-oscillation is almost the same as that of free oscillation. Also, no significant differences are found between aluminum and NC nylon.

The change of the oscillation amplitudes is shown in Fig. 9c, as a function of the oscillation period. The peak amplitudes, shown with circular markers, are almost the same for aluminum and nylon, and have a linear relationship with the oscillation period. Contrarily, the amplitudes at $200 \mathrm{~s}$, which are shown with cross markers, are considerably different for the two materials. Whereas aluminum cylinder kept almost the same amplitude, the amplitude was damped for the nylon cylinder. Especially when the period, as well as the amplitude, were small, the nylon cylinder stopped the oscillation due to overheating. The results indicate that the self-induced oscillation can continue for long time when the material of cylinder is highly heat-conductive.

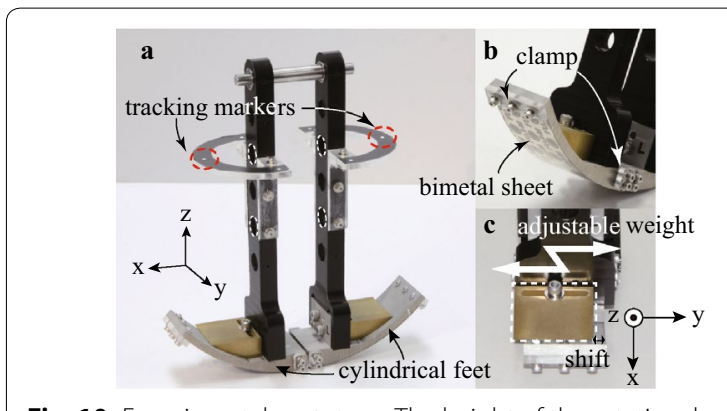

Fig. 10 Experimental prototype. The height of the rotational axis $h_{r}$ and the position of the weight on the feet are adjustable 


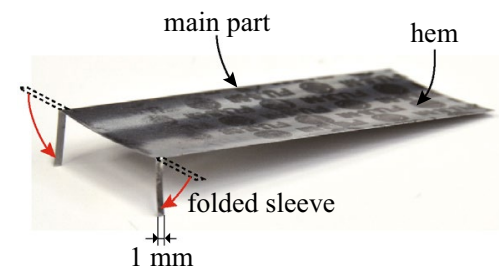

Fig. 11 T-shaped bimetal sheet
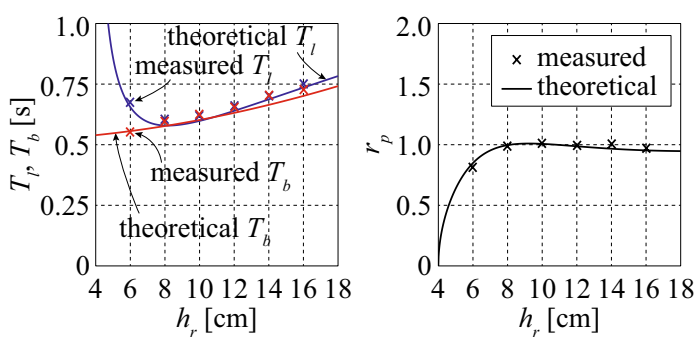

Fig. 12 Theoretical and measured periods, and their ratio

\section{Experiments \\ Prototype}

By following the proposed design process, and with aluminum feet, a new thermal walker was prototyped as shown in Fig. 10. The height $L$ is about $17 \mathrm{~cm}$, and the total weight $m$ is $310 \mathrm{~g}$. Each leg is composed of a thigh and a foot with an adjustable weight block. The weight of each leg $m_{l}$ is $145 \mathrm{~g}$ and the height of CoM of leg $h_{l}$ is $4 \mathrm{~cm}$. The thighs are made of polyacetal (black parts in the figure). At the bottom of thighs, 3-cm-wide aluminum feet are fixed. When both feet are aligned as in Fig. 10a, the bottom of the feet comprises a part of cylindrical surface, whose radius $R$ is $9 \mathrm{~cm}$. The two thighs are connected using a rotational axis and ball bearings. Each thigh has 6 holes at different heights for the bearings, such that $h_{r}$ can be changed for performance comparison. The rotational axis weighs $20 \mathrm{~g}$ and its length is $64 \mathrm{~mm}$. When the legs are fixed on the axis, their distance $d$ is $35 \mathrm{~mm}$. In the middle of each thigh, there is a black half-ring with a white tracking marker for optical motion tracking.

On the bottom of the feet, bimetal sheets (Fuji bimetal corp, $\mathrm{M}_{4}, 0.05-\mathrm{mm}$ thick, curvature coefficient: $13.7 \times 10^{-6} \mathrm{~K}^{-1}$ ) are fixed. Each sheet is shaped like a T-shirt, as in Fig. 11 and fixed by clamps at its sleeves and hem as shown in Fig. 10b. On the top surfaces of the feet, brass blocks are fixed as weights, which weigh $45 \mathrm{~g}$ each. Figure 10c shows a side view of the foot and the brass weight. The brass weight is shifted backward (which is leftward in the figure) to shift the CoM of the legs. Due to the backward shift, the prototype is expected to walk in the positive y direction because this shift induces forward swing in the positive y direction while the foot is in the air.

\section{Period ratio}

We measured the swing periods $T_{b}$ and $T_{l}$, and their ratio $r_{p}$, for different $h_{r}$ by changing the fixing holes for the rotational axis. The period of the leg swing was measured by rigidly fixing one of the legs upright and by treating the other leg as a pendulum. The period of the body swing was measured without the shift of the leg weight, such that the walker body behaves as a simple eccentric cylinder. The measured results and the theoretical estimations using the simplified leg model are shown in Fig. 12. As seen in the plots, the measured results agreed with the theoretical estimation. In the design process described above, $h_{r}$ was chosen as $12 \mathrm{~cm}$. The plot indicates that $r_{p}$ can be kept almost constant when $h_{r}$ is around $12 \mathrm{~cm}$.

\section{Experiment on heated horizontal surface}

Walking experiment on a hot horizontal surface was conducted using the developed prototype. Figure 13 shows sequential snapshots of the walker during one body swing, taken from above. The snapshots were cut out from a movie taken using an SLR camera (Nikon, D7000) with $24 \mathrm{fps}$. The height of the rotational axis $h_{r}$ was adjusted to $16 \mathrm{~cm}$, for this particular movie. The shift of the brass weight on the feet was $2 \mathrm{~mm}$. The temperature of the horizontal surface was kept at $130^{\circ} \mathrm{C}$ by using a heater, and the room temperature was $22^{\circ} \mathrm{C}$. The walker progressed in y direction on the hot surface swinging its body, first in the negative $\mathrm{x}$ direction and then in the positive $x$ direction. A leg swung only forward while it was in the air; no backward leg swing was observed. As the backward leg swing occurs when the ratio is larger than 1 , we can conclude that the ratio was not larger than 1 for this prototype.

Next, the gait of the prototype was observed. The hot surface was kept horizontal by using a bubble level, whose resolution is $4 \mathrm{~s}$ of inclination. Three different surface temperatures were tested, which were 110, 130 and $150^{\circ} \mathrm{C}$. These temperatures are lower than the previous study [8], in which the temperature was $170^{\circ} \mathrm{C}$. The gait of the walker was recorded for $20 \mathrm{~s}$ by an overhead high speed camera (Keyence, VW-6000), as shown in Fig. 14. The shutter speed and the frame rate were $8 \mathrm{~ms}$ and $60 \mathrm{fps}$, respectively. By changing the height of the axis $h_{r}$ as well as the shift of the leg weights, the walking was observed. After the experiment, the 


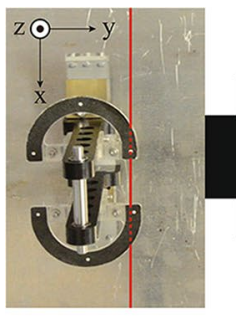

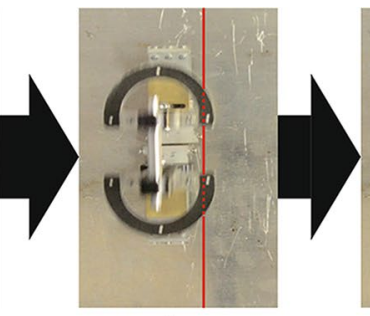

b

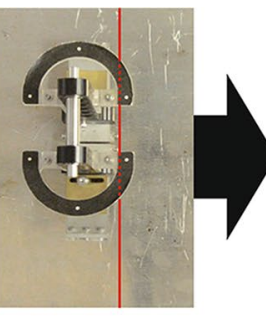

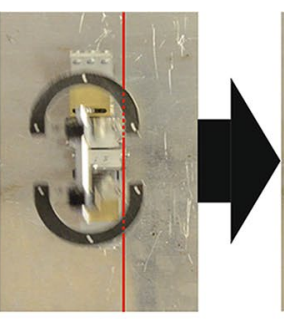

d

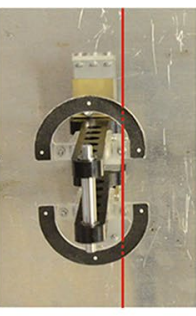

e

Fig. 13 Top view of the thermal walker walking on a heated horizontal surface. $L=16 \mathrm{~cm}$, weight shift $=2 \mathrm{~mm}$, heating temp. $=130^{\circ} \mathrm{C}$. $\mathbf{a} t=0 \mathrm{~s}$, $\mathbf{b}$ $\mathrm{t}=0.21 \mathrm{~s}, \mathbf{c t}=0.38 \mathrm{~s}, \mathbf{d} \mathrm{t}=0.58 \mathrm{~s}, \mathbf{e} \mathrm{t}=0.79 \mathrm{~s}$

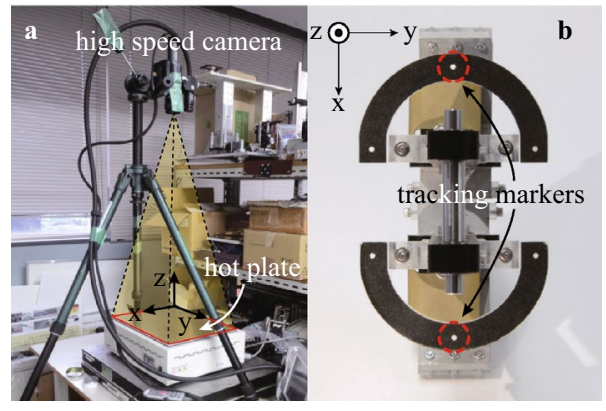

Fig. 14 Experimental setup and the view from the camera. The markers on the prototype were traced for gait observation

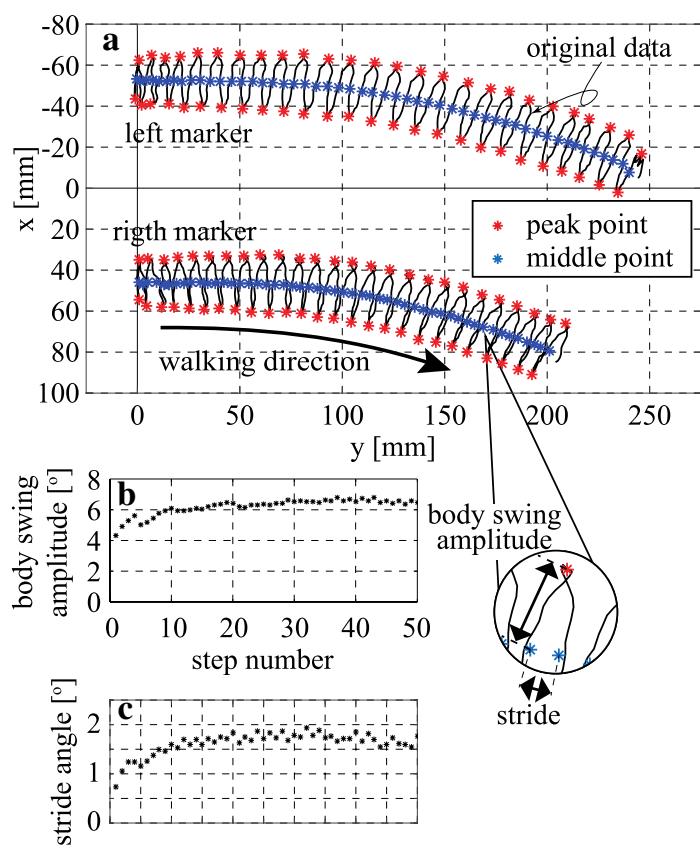

Fig. 15 Typical trajectories of the two markers and variations of body swing amplitude and stride angle $(L=16 \mathrm{~cm}$, weight shift $=2 \mathrm{~mm}$, heating temperature $=150^{\circ} \mathrm{C}$ ) recorded movies were analyzed to distill the trajectories of the tracking markers.

Figure 15a shows one of the observed trajectories for $20 \mathrm{~s}$. Two black lines show trajectories of the markers on the left and right legs. The prototype was placed at $y=0$ at the beginning, and then walked toward the positive $y$ direction by swinging its body in $\mathrm{x}$ direction. Blue points are the middle points (in time domain) of two adjacent red points. The blue points were used for calculating the stride. The red points were used to calculate the amplitude of the body swing. Figure 15b, c show one example of the calculated body swing amplitudes and the strides, which are averages of the left leg and the right leg. The plots show that, in the beginning, a small body swing was excited which then gradually increased within 10 steps. The stride was also small in the beginning. But as the body swing grew, the stride also grew up synchronously, to reach the steady state at around the 20th step. The steady state values are around $6^{\circ}$ for the body swing amplitude, and $1.5^{\circ}$ for the stride angle. In the other conditions, the walking gait also reached steady-states after 20 steps.

By using the data after the 20th step, the average and deviation of the amplitude and stride for all conditions were calculated as shown in (Fig. 16). Figure 16a through $\mathrm{c}$ show the relation between the shift of the foot weights and the body swing amplitude. It was found that the body swing amplitude had negative correlation with the weight shift. It was also found that the amplitude depended on $h_{r}$; larger body swing amplitude was achieved when $h_{r}$ was large. In the design process described above, these parameters were not supposed to affect the body swing, as we assumed that the two swings can be decoupled. The results, however, implied that the two swings were actually coupled, although the effect of the coupling on the period ratio $r_{p}$ would not be so large.

Figure $16 \mathrm{~d}-\mathrm{f}$ show the corresponding stride angle. It is indicated that stride angle has a positive correlation 

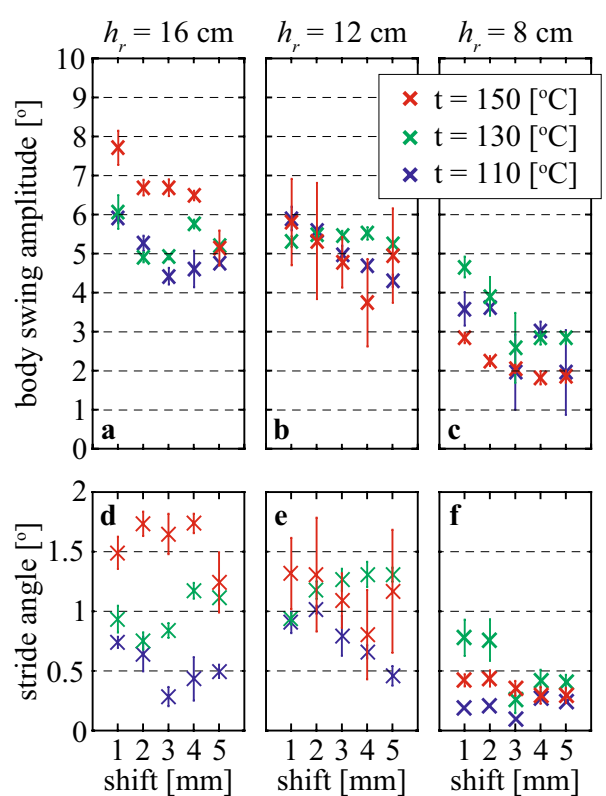

Fig. 16 Body swing amplitude $(\mathbf{a}-\mathbf{c})$ and stride $(\mathbf{d}-\mathbf{f})$ against the shift of the weight, with different $h_{r}$

with the body swing amplitude, although the results are scattered for $h_{r}$ of $16 \mathrm{~cm}$. Generally, larger weight shift resulted in smaller stride angle, which disagrees with the discussion in the previous study [8]. This might be explained by considering feet scuffing. The previous study assumed the length of the feet (in the walking direction $y$ ) was zero. Under such assumption, the study predicted that the stride would be proportional to the shift of CoM. This assumption might hold when the swing angles are large enough, as in the previous study. However, in this study, the swing angles were much smaller to realize $r_{p}$ of 1 , which would be the reason for the contradicting results. This aspect needs to be further analyzed in future studies.

\section{Conclusion}

In this paper, a new thermal walker was designed to realize simple walking, in which each leg swings forward only once in one body swing. A design process to realize such walking has been discussed using a simplified model. The developed prototype successfully realized the simple walking motion on a hot horizontal surface heated at $110^{\circ} \mathrm{C}$ or greater, in the ambient temperature of $22^{\circ} \mathrm{C}$. This temperature difference is lower than that of the previous study [8] by around $60^{\circ} \mathrm{C}$.

The temperature difference, however, is still too high for practical applications, and the future studies will aim at lowering the required temperature. One of the possible ways to reduce the temperature difference is to improve the fixation of the bimetal sheet for better contact with the feet. Since the heat in the bimetal is dissipated to the metallic feet, better contact between the bimetal and the feet will help the heat dissipation and reduce the requirement for the temperature difference. Or, amplifying the body swing by using a mechanical resonance would be also effective to decrease the requirement. If the difference between the required temperature and the ambient temperature can be smaller than, e.g., $30^{\circ} \mathrm{C}$, the walker would be able to walk on a hot ground during summer time.

\section{Authors' contributions}

TN contributed to the modeling, analysis, experiments, and drafting of the manuscript. AY took part in the conception, interpretation of data, and revising of the manuscript. Both authors read and approved the final manuscript.

\section{Competing interests}

The authors declare that they have no competing interests.

\section{Publisher's Note}

Springer Nature remains neutral with regard to jurisdictional claims in published maps and institutional affiliations.

Received: 19 January 2018 Accepted: 2 July 2018

Published online: 16 July 2018

\section{References}

1. McGeer T (1990) Passive dynamic walking. Int J Robot Res 9(2):62-82

2. McGeer T (1990) Passive walking with knees. In: Proceedings, 1990 IEEE international conference on robotics and automation, pp 1640-1645

3. Tedrake R, Zhang TW, Fong M, Seung HS (2004) Actuating a simple 3d passive dynamic walker. In: Proceedings ICRA'04, 2004 IEEE international conference on robotics and automation, vol. 5, pp 4656-4661

4. Collins SH, Ruina A (2005) A bipedal walking robot with efficient and human-like gait. In: ICRA 2005. Proceedings of the 2005 IEEE international conference on robotics and automation, pp 1983-1988. https://doi. org/10.1109/ROBOT.2005.1570404

5. Wisse M, Feliksdal G, Frankkenhuyzen JV, Moyer B (2007) Passive-based walking robot. IEEE Robot Automat Mag 14(2):52-62. https://doi. org/10.1109/MRA.2007.380639

6. Bhounsule PA, Cortell J, Grewal A, Hendriksen B, Karssen JD, Paul C, Ruina A (2014) Low-bandwidth reflex-based control for lower power walking: $65 \mathrm{~km}$ on a single battery charge. Int J Robot Res 33(10):1305-1321

7. Alghooneh M, Wu CQ, Esfandiari M (2016) A passive-based physical bipedal robot with a dynamic and energy-efficient gait on the flat ground. IEEE/ASME Trans Mechatron 21(4):1977-1984

8. Nemoto T, Yamamoto A (2015) Thermobot: a bipedal walker driven by constant heating. In: IEEE/RSJ international conference on intelligent robots and systems (IROS), pp 983-988

9. Harada Y, Suehara T, Ueno T, Higuchi T (2006) Development of actuator using thermal deformation. In: Proceedings of JSPE semestrial meeting 2006 spring, pp 803-804 (in Japanese)

10. Andronov AA, Vitt AA, Khaikin SE (1996) Theory of oscillators. Pergamon Press, Pergamon

11. Suehara T, Harada Y, Ueno T, Higuchi T (2006) Development of a selfpropelled system using thermal deformation. In: Proceedings of JSPE Semestrial Meeting, pp 805-806 (in Japanese)

12. Higuchi T, Ueno T (2010) Next-generation actuators leading breakthroughs. In: Higuchi T, Suzumori K, Tadokoro S (eds) Development of new actuators for special environment. Springer, London, pp 351-362 